\title{
Curve Adjustment Method: Deriving a Formula from a Graph
}

\author{
Jose Mujica \\ AES Fellow \\ Ramon A. Mata-Toledo \\ James Madison University
}

Audio Engineering, and others technical fields, need more mathematical-based methodologies. There are procedures in studio or live recordings that have not been tabulated yet and depend entirely on human experience and appreciation. Audio engineers whether at studios or live-music settings need tools that allow them to translate their daily personal annotations, rules of thumb, live-music and/or empirical experiments into mathematical expressions or formulas that can benefit the practitioners to get rid of the dependency on the personal and individual appreciation. In this paper, the authors attempt to describe a general procedure to convert any group of into a graph.

\section{INTRODUCTION}

In this article, the authors propose the use of the Least Square Method (LSM) as a procedure that can help us obtain a mathematical formula that can be used to complement, and if possible, better any human experience, particularly, on the area of sound quality. The LSM will be used to fit the curve of a graph from which we can generate a mathematical formula.

The thrust of the article is to help understand that an audio specialist does not need to be a mathematician to make use of a formula that can be easily derived from a graph. The procedure is a simple three-step process: first, collect data from his experiences on his field. Second, make a graph from the data obtained, and, as the third and last step, use the LSM to generate a mathematical formula. For example, you can determine a formula from your use of a dynamic processor such as a compressor. Suppose you have developed a method where you set the controls of the processor using a real-time spectrum analyzer. If you have set the controls to a sound that have particular amplitudes in three frequencies, then you can make a table of two variables for each group of musicians or songs. This table will help us plot each knob value against its corresponding sound with the same three frequency amplitudes. From the plot of this graph, after applying the LSM, we can generate a formula that can be easily taught and used worldwide without the need of having specialized workshops to teach the use and application of the formula. 


\section{USING THE LEAST SQUARE METHOD TO FIND THE EQUATION OF THE CEILING OF A ROOM}

In this paper, to illustrate the proposed methodology in a step-by-step fashion, we will use a real-life example to find the equation of the curve of a room's irregular lateral roof. The objective is to obtain a graph to which we can apply the LSM to derive a mathematical formula that we can use, as indicated later, for several other purposes including the improvement of the quality sound of the room. As a caveat to the reader, the figures used in this paper are not at a true scale although the values shown are actual measurements; all figures are only used to convey shapes and procedures. Figure 1 illustrates the irregular-room's profile and its dimensions in meters.

FIGURE 1 SIDE VIEW OF THE ROOM
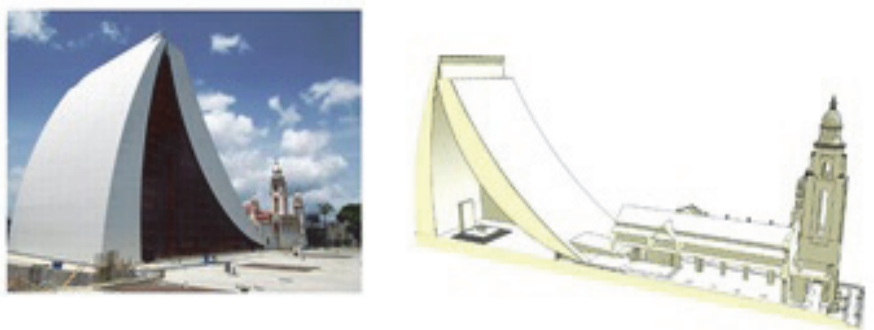

As it can be observed from Figure 1, the shape of the ceiling is convex upward. The challenge here is that, for this type of ceiling, there is not a specific formula to calculate the area under the curve or the area of the side walls. These areas may be needed for several purposes such as to calculate the amount of acoustic material or paint necessary to cover the walls or to determine the volume of the room for other acoustic applications.

As mentioned before, the authors propose the use of the LSM to obtain the applicable mathematical formula that allow us to plot the ceiling's profile and from it derive a mathematical formula. [1].

\section{Identifying the Coordinates}

The coordinates of the graph were found by measuring the height of the ceiling at seven different horizontal intervals including the height at the end of the curved wall as shown in Figure 2.

\section{FIGURE 2 \\ HEIGHT OF THE CEILING AT A DIFFERENT SPACE INTERVALS}

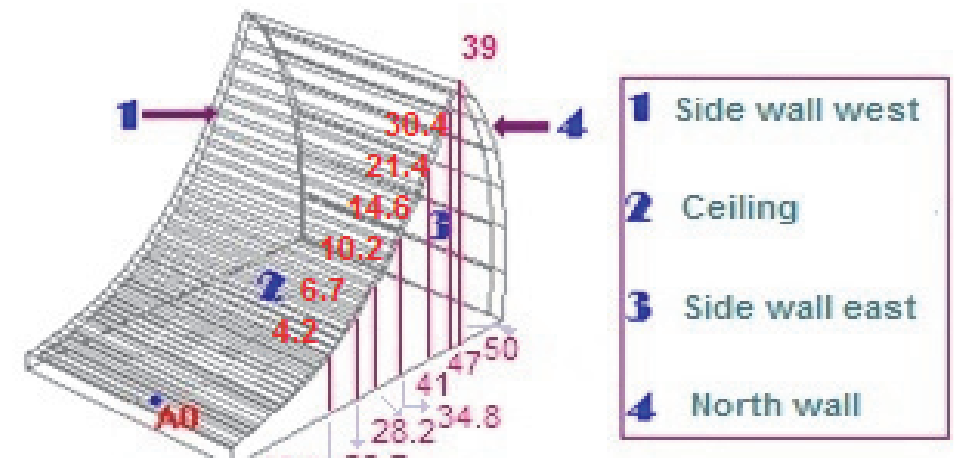




\section{Choosing the Curve's Form}

After determining the coordinates, it was necessary to choose a curve that approximates the shape of the room's ceiling. Figure 3 shows the graphs of some of the most commonly used functions for fitting curves using the LSM. [2]

FIGURE 3

CURVES OF COMMONLY USED FUNCTIONS

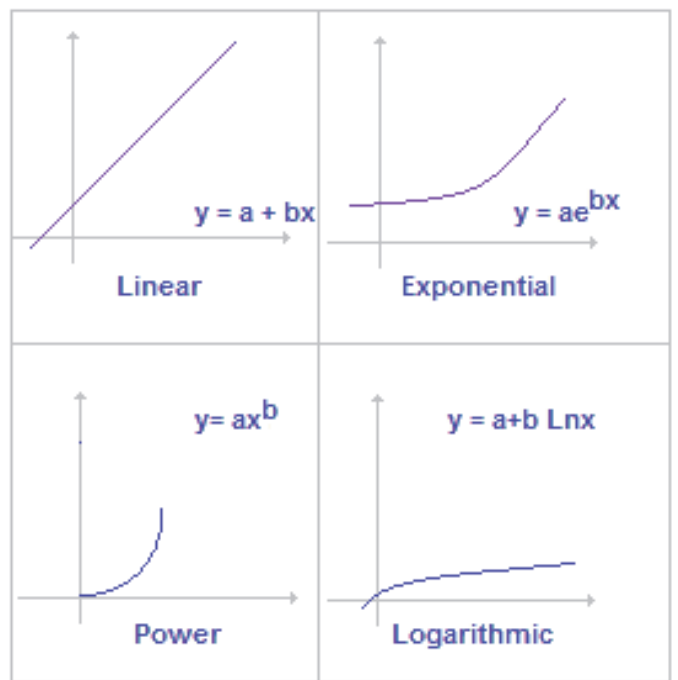

For this case, we chose the graph of the exponential function

$\mathrm{y}=\mathbf{a} e^{\mathbf{b x}}$

because its mirror-image, about the horizontal axis of the graph, seems to approximate best the convexdownward shape of the roof as shown in Figure 4.[3] We will use the LSM to fit the exponential graph to the profile of the roof.

FIGURE 4

FITTING THE CURVE'S LATERAL VIEW

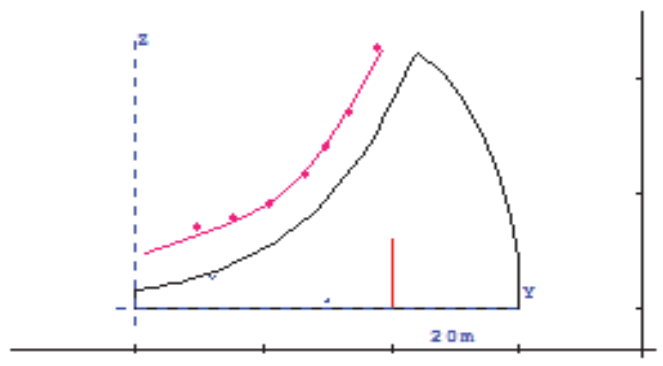

\section{SYSTEM OF EQUATIONS}

To obtain the coefficients $\mathbf{a}$ and $\mathbf{b}$ of the exponential function, LSM requires solving the system of equations (2) shown below. Table I explains the meaning of the different terms depending on the type of curve selected. 
$\mathrm{An}+\mathrm{B} \sum \mathrm{X}_{\mathrm{i}}=\sum \mathrm{Y}_{\mathrm{i}}$

$\mathrm{A} \sum \mathrm{X}_{\mathrm{i}}+\mathrm{B} \sum \mathrm{X}_{\mathrm{i}^{2}}=\sum \mathrm{X}_{\mathrm{i}} \mathrm{Y}_{\mathrm{i}}$

TABLE 1

VALUES OF THE SYSTEM EQUATION ELEMENTS

\begin{tabular}{|l|l|l|l|l|}
\hline CURVE & A & B & Xi & Yi \\
\hline Linear & a & b & xi & yi \\
\hline Exponential & Ln(a) & b & xi & Ln(yi) \\
\hline Power & a & b & Ln(xi) & yi \\
\hline Logarithmic & Ln(a) & b & Ln(xi) & Ln(yi) \\
\hline
\end{tabular}

\section{Rewriting the System}

Substituting the exponential values into (2) we obtain

$\mathrm{n} \operatorname{Ln}(\mathrm{a})+\mathrm{b} \sum \mathrm{x}=\sum \operatorname{Ln}(\mathrm{y})$

$\operatorname{Ln}(\mathrm{a}) \sum \mathrm{x}+\mathrm{b} \sum \mathrm{x}^{2}=\sum \mathrm{x} \operatorname{Ln}(\mathrm{y})$

where " $n$ " is the number of coordinates previously obtained and whose values are shown in Table 2.

TABLE 2

\section{ROOF COORDINATES}

\begin{tabular}{|c|c|}
\hline $\mathrm{X}$ & $\mathrm{Y}$ \\
\hline 17.3 & 4.2 \\
\hline 22.7 & 6.7 \\
\hline 28.2 & 10.2 \\
\hline 34.8 & 14.6 \\
\hline 41 & 21.4 \\
\hline 47 & 30.4 \\
\hline 50 & 39 \\
\hline
\end{tabular}

\section{SOLVING THE SYSTEM OF EQUATIONS}

\section{Calculating the Individual Terms}

In this example, $\mathrm{n}=7$ (the number of coordinates)

The summation of all Xs values is:

$$
\sum x=17.3+22.7+28.2+34.8+41+47+50=241
$$

The summation of all the squares of the Ys coordinates is:

$$
\sum x^{2}=299.29+515.29+795.24+1211.04+1681+2209+2500=9210.86
$$

The summation of the logarithms of the Ys coordinates of Table II is:

$$
\sum \operatorname{Ln}(y)=\operatorname{Ln}(4.2)+\operatorname{Ln}(6.7)+\operatorname{Ln}(10.2)+\operatorname{Ln}(14.6)+\operatorname{Ln}(21.4)+\operatorname{Ln}(30.4)+\operatorname{Ln}(39)=18.5
$$




$$
\sum \operatorname{Ln}(y)=1.44+1.9+2.3+2.68+3.1+3.43+3.67=18.5
$$

The summation of all Xs coordinates multiplied by its corresponding Y logarithm is:

$$
\begin{gathered}
\sum x \operatorname{Ln}(y)=17.3 * 1.44+22.7 * 1.9+28.2 * 2.3+34.8 * 2.68+41 * 3.1+47 * 3.43+50 * 3.67=697.98 \\
\\
\sum x \operatorname{Ln}(y)=4.91+43.1+64.86+93.26+127.1+161.2+183.5=697.98
\end{gathered}
$$

Substituting All Terms in the System of Equation (3)

Performing the corresponding arithmetic operations, we obtain the following results:

7.Ln(a) $+241 . b=18.5$

241. $\operatorname{Ln}(\mathrm{a})+9210.86 . \mathrm{b}=697.98$

\section{CALCULATING THE b COEFFICIENT}

Solving the top equation of (4) for $\operatorname{Ln}($ a) give us

$$
\operatorname{Ln}(\mathrm{a}) \approx \frac{18.5-241 \mathrm{~b}}{7}
$$

Replacing this value in the second equation of (4) we obtain:

$$
241\left(\frac{18.5-241 b}{7}\right)+9219.86 b=697.98
$$

Solving for the coefficient $\mathbf{b}$ from the latter equation results in

$$
\boldsymbol{b}=\frac{61.02}{913.23} \approx 0.067
$$

\section{CALCULATING THE a COEFFICIENT}

To find the coefficient $\mathbf{a}$, we replace the value of $\mathbf{b}$ in the second equation of the system (4) and solve for $\operatorname{Ln}(\mathrm{a})$. That is,

$$
\begin{gathered}
\text { 241. } \operatorname{Ln}(a)+9210.86 . b=697.98 \text { where } b \approx 0.067 \\
241 . \operatorname{Ln}(a)+9210.86 .(0.067)=697.98 \\
241 \operatorname{Ln}(a)=697.98-9210.86(0.067) \\
241 . \operatorname{Ln}(a)=697.98-617.13 \\
\operatorname{Ln}(a)=\frac{80.9}{241} \cong 0.336
\end{gathered}
$$

Raising e to value just obtained results in:

$$
e^{\operatorname{Ln}(a)}=e^{0.336} \rightarrow a=e^{0.336} \rightarrow \mathbf{a} \approx 1.4
$$




\section{EQUATION OF CEILING'S CURVE}

Following the procedure outlined we can rewrite the equation (1) using the coefficients a and $\mathbf{b}$ just calculated

$$
\begin{gathered}
\mathbf{a} \approx 1.4 \\
\mathbf{b} \approx 0.067
\end{gathered}
$$

Using the exponential equation previously selected (1)

$$
y=\mathbf{a e}^{\mathbf{b x}}
$$

and substituting the coefficients, $\mathbf{a}$ and $\mathbf{b}$ we get the following function

$y=1.4 e^{0.67 x}$

When we plot the latter equation into the Maple software the values of $x$ initially obtained in table 2, the graphic (See Figure 5 below) obtained is very close to shape of the Figure 1. So, equation (I) approximates indeed the profile of the room ceiling.

$$
\text { expr }:=\left\{\begin{array}{cc}
1.4 \mathrm{e}^{(.067 x)} & x \leq 49.5 \\
0 & \text { otherwise }
\end{array}\right.
$$

FIGURE 5

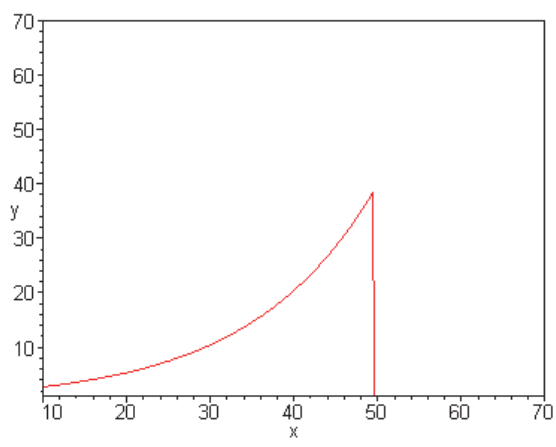

Now that we have a formula for the curve for the profile of the roof, we can calculate the area of the wall by calculating a definite integral between the curve, the two points representing the length of the room (0 and 54) and the floor limits. The integral can be solved using different applications some of which are available free of charge in the Internet.

\section{CONCLUSION}

The real-life example of this paper highlights that the use of the Least-Squares for fitting curves may be a powerful tool for Audio Engineers. The authors hope that the procedure illustrated above can help the practitioner and improve the human appreciation of audio engineers' experiences which, when converted into formulas, can help them fine tune their skills. In addition, to this specific real-life example, LSM has been useful for Reverb Time Tables to evaluate its effect in longer rooms, cable Vs. electrical resistance tables related to the gauge; the Acoustics Mass Law and some other acoustic applications. LSM can also be useful in other fields where it can help quantify the human appreciation of the physical world. 
It is important to observe that the use of the LSM is not a panacea for improving quality sound nor that it always produce the best fit. However, we make emphasis on it on this paper because its simplicity and wide range of applications. In addition to the MLS, there are other methods for fitting curves each one with its own advantages and disadvantages. With practice, you will learn that there are curves that, in some specific situation, may fit better than others, for instance, in some occasions, a power function may fit better than an exponential. Fitting a curve is an iterative process which may need to be tried out with several different curves. The adage "practice makes perfect" applies to finding the best fit to determine mathematical formulas which can help improve the human perception.

\section{REFERENCES}

Hewlett-Packard, Standard Applications Pac HP-4C, 00041-90018 Rev D 1980. http://www.hp41.org/LibView.cfm?Command=View\&ItemID=376

Kolb, W. M. (1982). Curve Fitting for programmable calculators, First Edition. William M. Kolb, 34 Laughton Street, Upper Marlboro, Maryland 20772, USA.

Maple Software plot. (n.d,). Retrieved from https://www.maplesoft.com/

McCracken, D. D., \& Dorn, W. S. (1964). Numerical Methods and Fortran Programming, $1^{\text {st }}$ Edition, John Wiley \& Sons Ltd.

Mujica, J, (1982). Infinitesimal Calculus with Analytic Geometry and Computing Applied to Audio, First Edition. In Spanish, Editorial Oasis, Venezuela.

Struck, C. (2010, November). ZFIT: A MATLAB Tool for Thiele-Small Parameter Fitting and Optimization. CJS Labs, San Francisco, CA, USA, Audio Engineering Society Convention 129. Retrieved from http://www.aes.org/e-lib/browse.cfm?elib=15642 